\title{
3D Printable Lightweight Porous Superstrate for Improved Radiation Performance of Antenna
}

\author{
Touseef Hayat*, Muhammad U. Afzal*, Foez Ahmed*, Ali Lalbakhsh*, and Karu P. Esselle ${ }^{\dagger}$ \\ * Macquarie University, Sydney, Australia \\ $\dagger$ University of Technology Sydney, Sydney, Australia \\ touseef.hayat@students.mq.edu.au
}

\begin{abstract}
The paper presents a 3D printable porous superstrate (PS) to enhance directive radiation performance of low to medium gain antennas. The PS design process is based on theory of near-field phase correction. Transmission phase through PS is locally varied by changing sizes of perforations in different sections of the PS. The PS is designed for a resonant cavity antenna (RCA) using acrylonitrile butadiene styrene (ABS) filament. With PS the RCA aperture phase is relatively planar and its directivity in boresight direction is increased by $7.2 \mathrm{~dB}$ $(14.804 \mathrm{~dB}$ to $22 \mathrm{~dB})$ along with $8.2 \mathrm{~dB}$ reduction in side-lobe levels (SLL) and $31 \%$ increase in aperture efficiency.

Index Terms-additive manufacturing, directivity enhancement, perforated dielectric, phase correction, rapid prototyping.
\end{abstract}

\section{INTRODUCTION}

Additive manufacturing a.k.a 3D printing is a cost-effective process to synthesize three-dimentional structures by depositing successive layers of materials through computer control. Using the 3D-printing method, internal features such as air voids can be implanted in three dimensional models, with submillimeter range accuracy. Moreover, prototypes can be rapidly fabricated, which makes this method predominantly advantageous for high value but low volume designs in radio frequency (RF) regime. Numerous RF components such as frequency selective surfaces (FSS), lenses and waveguides have been fabricated using AM, which is cost-effective and offers extra design freedom compared to conventional techniques [1], [2], [3], [4]. This paper presents preliminary results of lowcost and lightweight 3D printable PS comprising of cylindrical perforations to locally implement phase transformation.

Introduction of air perforation in dielectric material is the simplest method to change its composition [3]. Change in composition of the dielectric material results in variation in its transmission characteristics. This concept can be used for aperture phase error rectification of antennas with non-uniform phase on the aperture plane. Where, distinct phase delay values are required in different sections to compensate the propagating phase shift experienced by the propagating wave. Dielectric unit-cells with particular perforation size can be introduced in appropriate spaces at aperture to attain uniform phase response. This yields planar low profile superstrates, which helps to avoid shadowing effect contrary to non-planar ones proposed in literature [2], [5].

\section{RCA CONFIGURATION}

The RCA above patch considered here, comprises of patch antenna and an octogonal shaped partially reflecting super- strate (PRS) as shown in Fig. 1. Design frequency of patch is $20 \mathrm{GHz}\left(\lambda_{o}=15 \mathrm{~mm}\right)$, where $\lambda_{o}$ is freespace wavelength. Patch is printed on Rogers UltraLam2000 substrate of thickness 1.57 mm, while PRS is made of Rogers TMM10 material with thickness $1.24 \mathrm{~mm}$. Maximum lateral dimension of PRS in xy-plane is $90 \mathrm{~mm}\left(6 \lambda_{o}\right)$ and the PRS is suspended at $\lambda_{o} / 2$ spacing. A hypothetical plane named input phase plane (I$\mathrm{PP}$ ) is defined $7.1 \mathrm{~mm}$ above the PRS to probe dominant Efield component radiated by RCA. Considering the circular symmetry of aperture phase, a method to probe and correct it by dividing in nine concentric circles with constant offset of $\lambda_{o} / 3$ is explained in [5]. Probed phase values at I-PP are shown in Fig. 2(a), which is symmetric around the center. The phase of the field in the middle is higher, hence the PS must have prowess to offer greater phase delay in the middle and vice-versa. Next section explains how phase delay is locally varied in the PS.

\section{PS DESIGN METhodology}

The basic building block of the PS is a cuboid dielectric of ABS material $\left(\epsilon_{r}=4.4\right)$, with lateral dimensions $5 \mathrm{~mm}\left(\lambda_{o} / 3\right)$ and height $17 \mathrm{~mm}$, as shown in Fig. 2(b). There is a hole or perforation in the block that controls the phase delay in

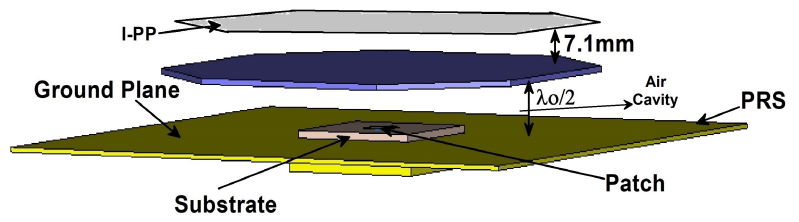

Fig. 1: Basic configuration of RCA with plane to probe aperture phase (I-PP).

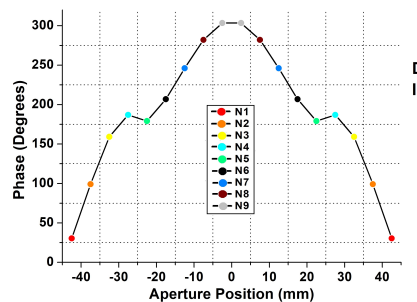

(a)

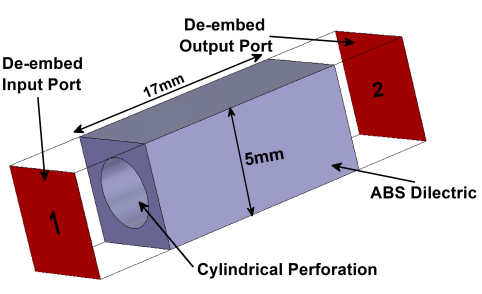

(b)
Fig. 2: (a) probed phase error at I-PP (b) Unit-cell configuration with cylindrical perforation. 
the PS. The block a.k.a unit-cell is simulated with periodic boundaries in CST Studio Suite and the transmission phase delay is recorded by varying the radius of perforation size between 0 and $1.8 \mathrm{~mm}$. Plot for variation in transmission phase delay through the unit-cell under consideration with change in perforation is given in Fig. 3(a). Transmission phase delay is maximum when there is no perforation at all and exponentially reduces with increase in perforation size. It is because of exponential rise of air component in the unit-cell with linear increase in perforation radius. This suggests that RCA's phase uniformity on the aperture can be improved by introducing cells with appropriate phase delay. Cells will be arranged in form of concentric circles at I-PP, which will lead to $17 \mathrm{~mm}$ high PS. Relatively planar phase is expected after field propagates through the PS. Table I lists error for half of the aperture recorded at I-PP. The normalized phase values and required phase delay values are also listed, which have been calculated by setting constant required phase value above PS, as explained in [5]. Corresponding perforation sizes that give required delay with respect to aperture position are also given. Simulated model of PS by arranging cells in circle is given in Fig. 3(b). The proposed low-cost model can be rapidly prototyped using household printer in single step by evading the traditional machining methods.

\section{RESUlts}

Simulated far-field directivity patterns of RCA in E and H-plane, with and without PS are shown in Fig. 4, which illustrates that the introduction of PS resulted in $7.2 \mathrm{~dB}$ enhancement in directivity and $8.2 \mathrm{~dB}$ reduction in SLL. The

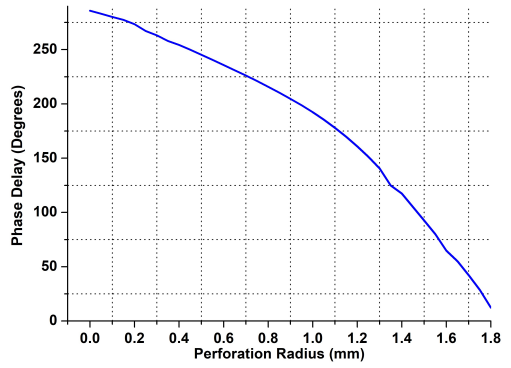

(a)

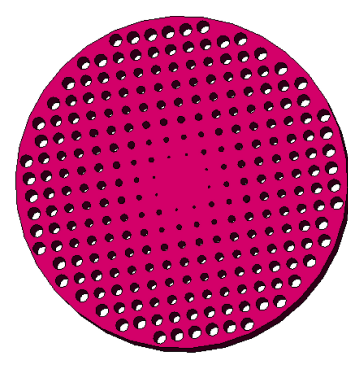

(b)
Fig. 3: (a) Effect of perforation size on transmission phase delay (b) Perspective view of PS.

TABLE I: Perforation radius and phase errors for PS

\begin{tabular}{|c|c|c|c|c|}
\hline $\begin{array}{c}\text { Position } \\
(\mathbf{m m})\end{array}$ & $\begin{array}{c}\text { Phase (I-PP) } \\
\text { (deg.) }\end{array}$ & $\begin{array}{c}\text { Normalized } \\
\text { phase (deg.) }\end{array}$ & $\begin{array}{c}\text { Required } \\
\text { phase (deg.) }\end{array}$ & $\begin{array}{c}\text { Perforation } \\
(\mathbf{m m})\end{array}$ \\
\hline 2.5 & 303.46 & 0 & 285.5 & 0 \\
\hline 7.5 & 282.06 & -21.4 & 264.1 & 0.3 \\
\hline 12.5 & 246.07 & -57.4 & 228.1 & 0.7 \\
\hline 17.5 & 206.81 & -96.6 & 188.8 & 1 \\
\hline 22.5 & 179.18 & -124.2 & 161.2 & 1.2 \\
\hline 27.5 & 186.98 & 116.5 & 169.0 & 1.15 \\
\hline 32.5 & 159.20 & -144.2 & 141.2 & 1.3 \\
\hline 37.5 & 99.18 & -204.3 & 81.2 & 1.55 \\
\hline 42.5 & 30.34 & -273.22 & 12.4 & 1.8 \\
\hline
\end{tabular}

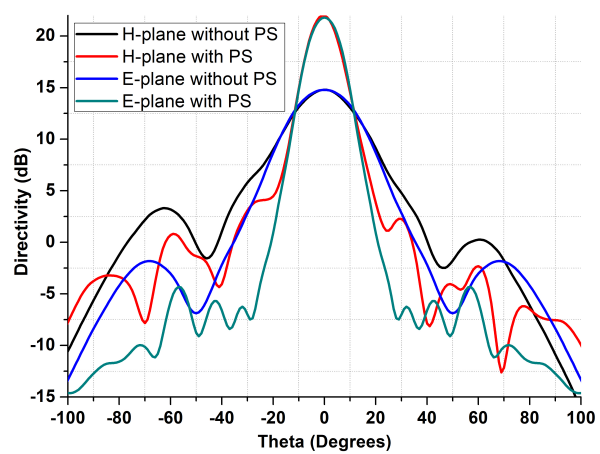

Fig. 4: Directivity response in E and H-planes with and without PS.

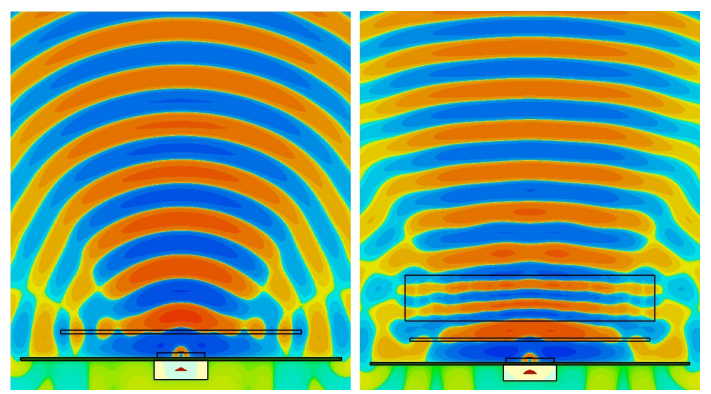

Fig. 5: E-Field response in H-plane (a) without PS (b) with PS.

snapshot of E-field propagating in a cross-section of RCA, with and without PS, is shown in Fig. 5. It is evident that with PS (right) the phasefronts are nearly planar and post processing indicated that maximum phase variation in a plane parallel to RCA aperture is reduced from $285.5^{\circ}$ to $43^{\circ}$. Simulation based gain calculations showed that with PS the aperture efficiency is $47 \%$ which is only $16 \%$ without it.

\section{REFERENCES}

[1] T. Hayat, M. U. Afzal, A. Lalbakhsh, and K. P. Esselle, "Additively Manufactured Perforated Superstrate to Improve Directive Radiation Characteristics of Electromagnetic Source," IEEE Access, vol. 7, pp. 153445 - 153452, oct 2019. [Online]. Available: https://ieeexplore.ieee.org/document/8878089/

[2] T. Hayat, K. P. Esselle, M. U. Afzal, and K. Singh, "3D Printed All Dielectric Phase Correcting Surface for Resonant Cavity Antenna," in Proceedings of the 2018 IEEE 7th Asia-Pacific Conference on Antennas and Propagation, APCAP 2018. Institute of Electrical and Electronics Engineers Inc., nov 2018, pp. 214-215.

[3] T. Hayat, M. U. Afzal, A. Lalbakhsh, and K. P. Esselle, "3-D-Printed Phase-Rectifying Transparent Superstrate for Resonant-Cavity Antenna,' IEEE Antennas and Wireless Propagation Letters, vol. 18, no. 7, pp. 1400-1404, jul 2019.

[4] S. Zhang, R. K. Arya, S. Pandey, Y. Vardaxoglou, W. Whittow, and R. Mittra, "3D-printed planar graded index lenses," IET Microwaves, Antennas and Propagation, vol. 10, no. 13, pp. 1411-1419, oct 2016.

[5] M. U. Afzal, K. P. Esselle, and B. A. Zeb, "Dielectric Phase-Correcting Structures for Electromagnetic Band Gap Resonator Antennas," IEEE Transactions on Antennas and Propagation, vol. 63, no. 8, pp. 33903399, aug 2015. 